\title{
Investigation of the Influence of Swallowing, Coughing and Vocalization on Heart Rate Varia- bility with Respiratory-phase Domain Analysis
}

\author{
K. Kotani' ${ }^{1}$, M. Tachibana ${ }^{2}$, K. Takamasu ${ }^{2}$ \\ 'Graduate School of Information Science and Technology, The University of Tokyo, Tokyo, Japan \\ ${ }^{2}$ Graduate School of Engineering, The University of Tokyo, Tokyo, Japan
}

\begin{abstract}
Summary
Objectives: The objective of our study is to investigate extrinsic influences on heart rate variability using respiratory-phase domain analysis. Swallowing, coughing and vocalization (reading aloud and conversation) are adopted as extrinsic influences.

Methods: In this study, an instantaneous R-R interval (RRI) is sampled at each $\pi / 10 \mathrm{rad}$ of the respiratory phase and the data is divided into three subsets: a) respiration with event, b) one respiration after the event, and c) normal respiration. Then the mean waveforms of respiratory sinus arrhythmia (RSA) are calculated and compared.

Results and Conclusions: It is found that swallowing induces tachycardia that recovers within one respiration. Coughing also induces tachycardia, but it does not recover within one respiration. Vocalization shortens the mean RRI, but the changing respiratory pattern due to vocalization has no statistically significant influence on the amplitude of RSA. Furthermore, it is found that the proposed method is effective for analyzing extrinsic influences on heart rate variability (HRV).
\end{abstract}

\section{Keywords}

Heart rate variability, respiratory phase, respiratory sinus arrhythmia

Methods Inf Med 2007; 46: 179-185

\section{Introduction}

Heart rate variability (HRV) has been well studied because of its clinical and physiological importance [1]. We have previously proposed a method to extract respiratory sinus arrhythmia (RSA), which is known as an index of cardiac vagal activity [1-3], as a waveform in the respiratory-phase domain $[4,5]$. In the case of paced respiration, the amplitude of RSA by this method has interchangeability with the commonly used power of $0.15-0.5 \mathrm{~Hz}$ by frequency analysis. Moreover, by selecting data that correctly reflect vagal activity breath-by-breath, the theoretical accuracy of the vagal activity estimated by respiratory-phase domain analysis rises, as confirmed by a mental arithmetic experiment [5].

The measurement of RSA has also been used in the field of human engineering, and it is expected to be used to evaluate the difficulty of tasks, to optimize working environments and to improve subjects' health. However, for the measurement of RSA in a real situation, such as deskwork, conversation or relaxation, some extrinsic influences disguise the results. Consequently, it is important to investigate the extrinsic influence on HRV and extracted RSA. Generally for the investigation of how HRV reflects extrinsic elements, time-separated experiments are conducted and their results are compared. But there is the possibility that conditions differ between experiments. Moreover, the short-term variability, induced by the event, cannot be evaluated because it is hidden by RSA, which is the short-term dominant variability.

The objectives of this paper are: a) to focus on swallowing, coughing and vocali- zation as extrinsic influences, because these behaviors are observed normally even during rest and in sitting positions and can be detected by the motion of the throat; and b) to analyze their influences on HRV in the respiratory-phase domain by extracting the stable waveform of RSA and then comparing it with that of normal respiration.

\section{Methods}

\subsection{Experimental Procedures}

We obtained R-R intervals (RRI) of the electro-cardiogram (AC-601, NihonKoden) by a sampling frequency of $1 \mathrm{kHz}$, and instantaneous lung volume (ILV) by sampling an elastic chest band (TR-755T, Nihon-Koden) at a rate of $100 \mathrm{~Hz}$. Further, we put an accelerometer (8304B2, Kistler) on the larynx and sampled the motion of the laryngeal prominence to detect the time of swallowing, coughing and vocalization.

Five experiments were performed in rest and sitting positions: two for swallowing, one for coughing, and two for vocalization. The experimental protocols and the number of subjects are summarized in Table 1. In Exp. 1-1, swallowing was allowed to be performed by the subject at free timing, while in Exp. 1-2, it was also allowed at free timing but only between inspiration and expiration. In Exp. 2, coughing was performed once a minute on purpose. In Exps. 3-1 and $3-2$, vocalization was performed during two $90 \mathrm{~s}$ sessions (110-200 s and 290-380 s). For vocalization, reading aloud and conver- 
sation were performed in Exps. 3-1 and 3-2, respectively. These experiments were arranged symmetrically in time in order to cancel the linear trend of RRI and RSA.

\subsection{Signal-processing Algorithm}

Figure 1 shows a diagram of the signalprocessing algorithm. First the data of RRI

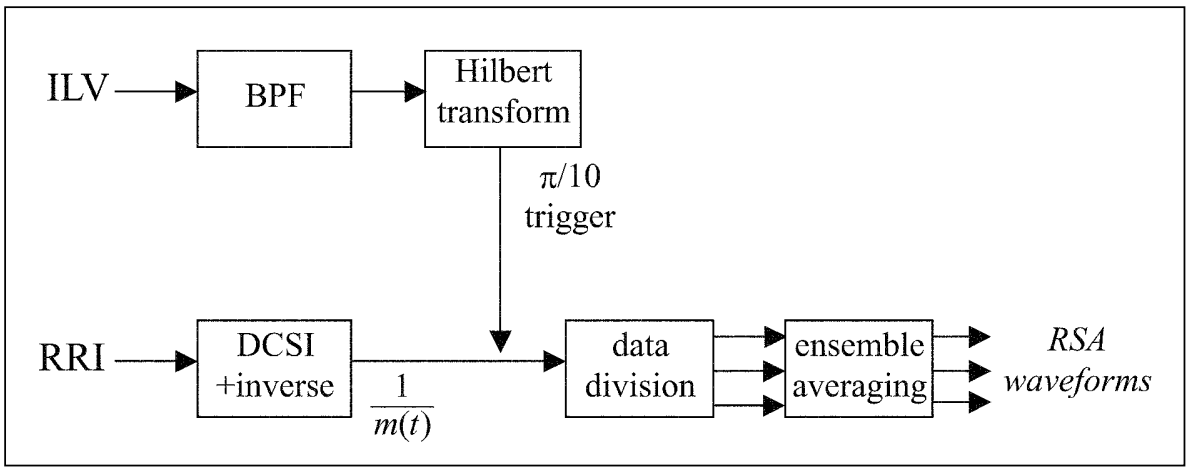

Fig. 1 Diagram of signal-processing algorithm was interpolated by the derivative of cubic spline interpolation (DCSI) [6] according to the following equation:

$M\left(t_{k}\right)=\int_{t_{0}}^{t_{k}} m(t) d t=k$,

where $t_{k}(k=0,1,2, \ldots, n)$ are times at which heartbeats are observed and $m(t)$ is the instantaneous heart rate. $m(t)$ is obtained from the first derivative of $M\left(t_{k}\right)$, which is constructed by the cubic spline interpolation. Here, we used $1 / m(t)$ as the instantaneous RRI. An instantaneous respiratory phase was estimated by analytic signal using a Hilbert transform [7] after filtering ILV. ILV was passed by a linear-phase finite impulse response band-pass filter whose pass-band was 0.1-10.0 Hz. Instantaneous RRI was resampled in each $\pi / 10 \mathrm{rad}$ of the respiratory

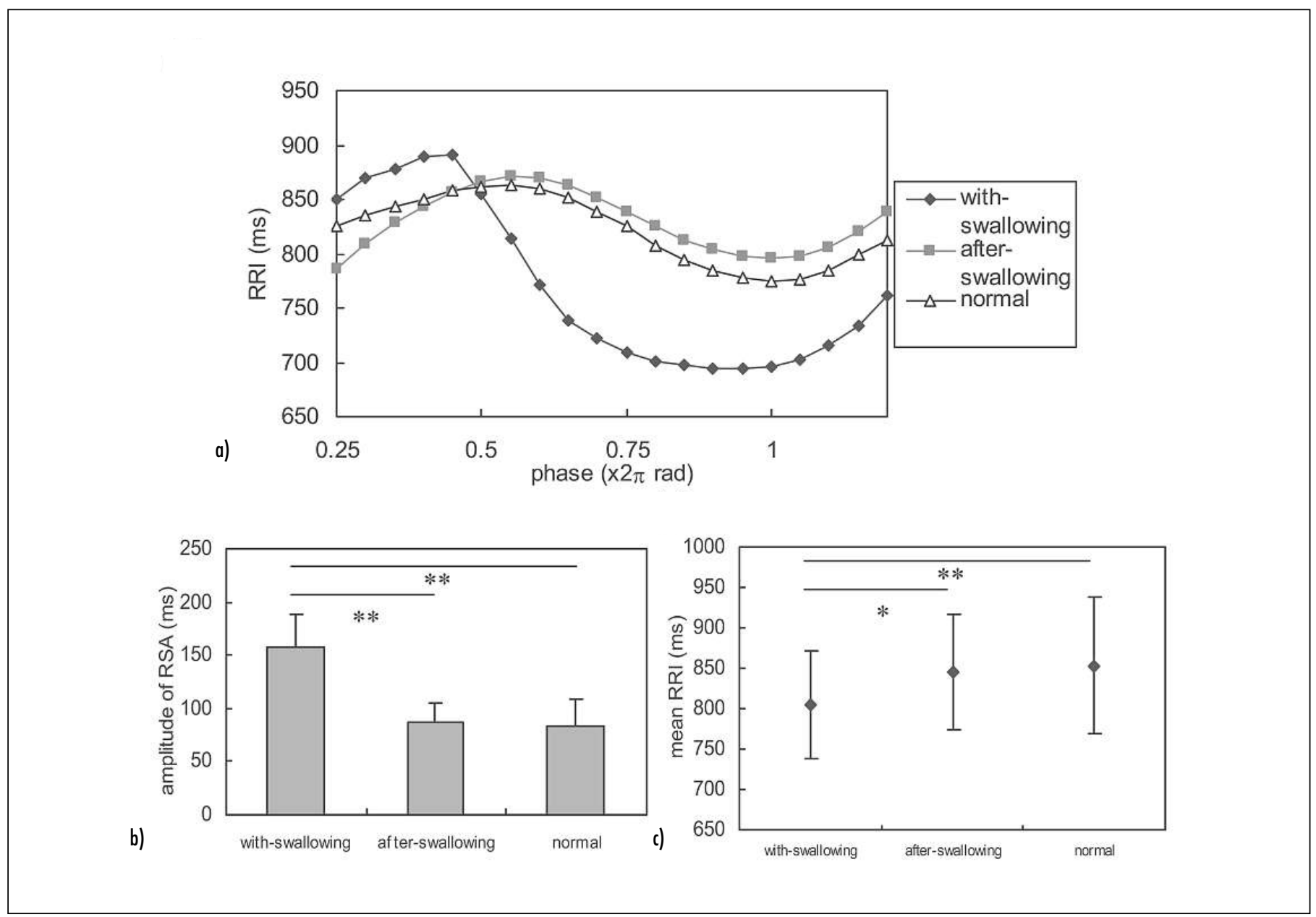

Fig. 2 Results of Exp. 1-1. a) RSA waveforms of a typical subject, b) RSA amplitudes of all subjects, c) mean RRIs of all subjects. Data are mean values with an error bar for standard deviation. ${ }^{* *}$ indicates $P<0.01$ and ${ }^{*}$ indicates $P<0.05$. 


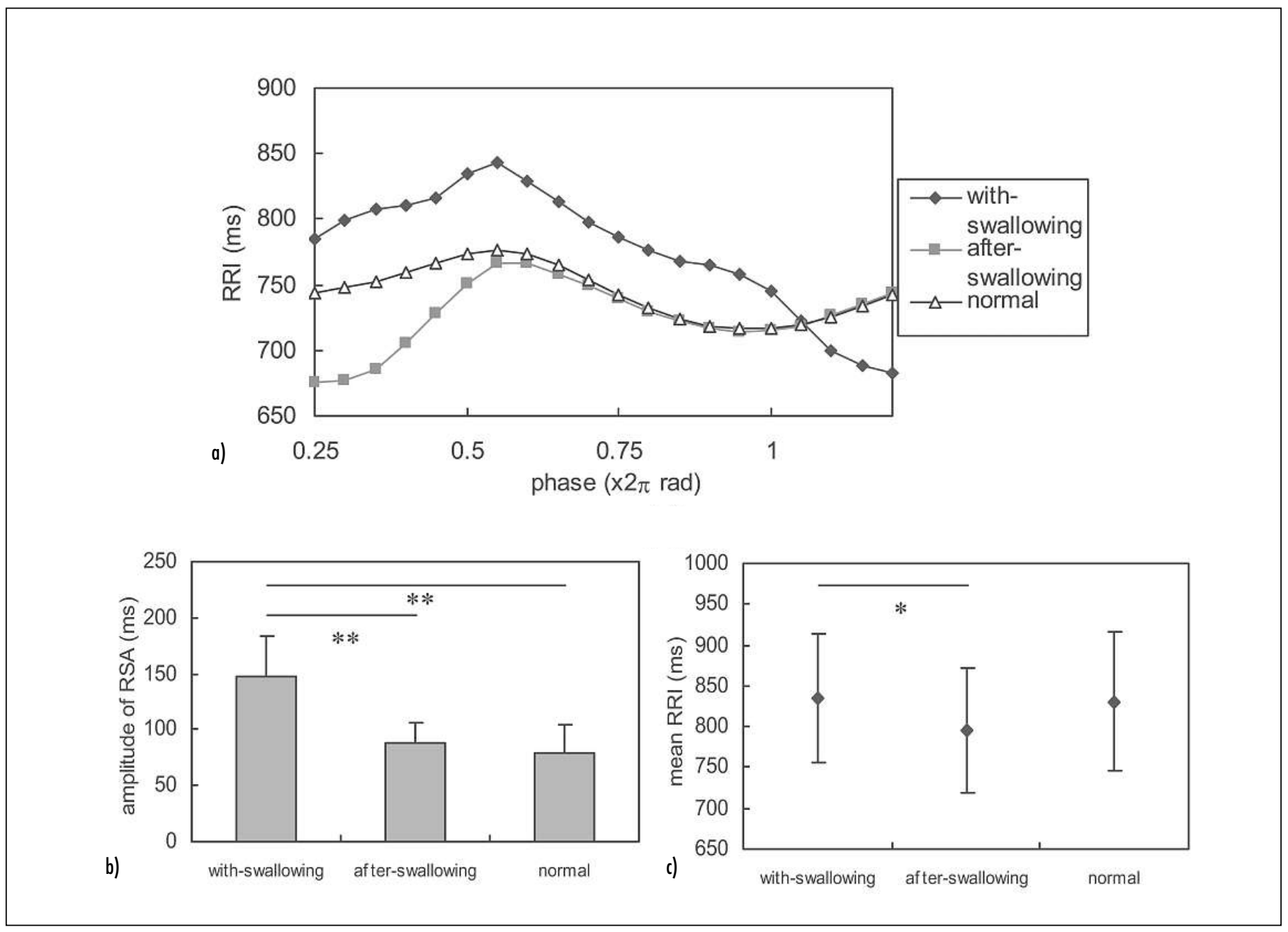

Fig. 3 Results of Exp. 1-2. a) RSA waveforms of a typical subject, b) RSA amplitudes of all subjects, c) mean RRIs of all subjects. ${ }^{* *}$ indicates $P<0.01$ and ${ }^{*}$ indicates $P<0.05$.

phase. We set the phase between $\pi / 2 \mathrm{rad}$ and the next $\pi / 2 \mathrm{rad}$ as one respiration, and both the inspiratory period (i.e. from $\pi \mathrm{rad}$ to $2 \pi$ $\mathrm{rad}$ ) and the tachycardia induced by inspiration were included in one respiration. The data was divided into respiration in which an event occurred, one respiration after the event, and normal respiration (in Exps. 3-1 and 3-2, one respiration after the event was not extracted). After that, in each subset, mean values of equi-sampled RRI in the same phase were calculated and stable waveforms of RSA were extracted. Then the waveforms of three (or two) subsets were compared.

In this method, the variability of RSA, which is the largest in the short-term fluctuation of HRV, can be distinguished by comparing these three (or two) waveforms. The variability of Mayer wave-related sinus arrhythmia and low-frequency fluctuation were minimized by ensemble averaging, and the influence of extrinsic elements was stabilized. By random event occurrence, it is expected that the influences of the low-frequency trend of RRI and RSA can be further minimized. It is another merit of this method that measurements are performed in natural conditions that do not require paced breathing.

For statistical analysis, mean RRI and the amplitude of RSA were calculated in each waveform. The amplitude of RSA was the

Table 1 Experimental protocols

\begin{tabular}{|l|l|l|l|l|}
\hline Experiment & Experimental condition & Measuring time $(s)$ & Timing & No. of subject \\
\hline $1-1$ & swallowing & 420 & free & 6 \\
\hline $1-2$ & swallowing after inspiration & 420 & free (after inspiration) & 6 \\
\hline 2 & coughing & 420 & once in a minute & 7 \\
\hline $3-1$ & reading aloud & 490 & two 90 s sessions & 7 \\
\hline $3-2$ & conversation & 490 & two 90 s sessions & 7 \\
\hline
\end{tabular}




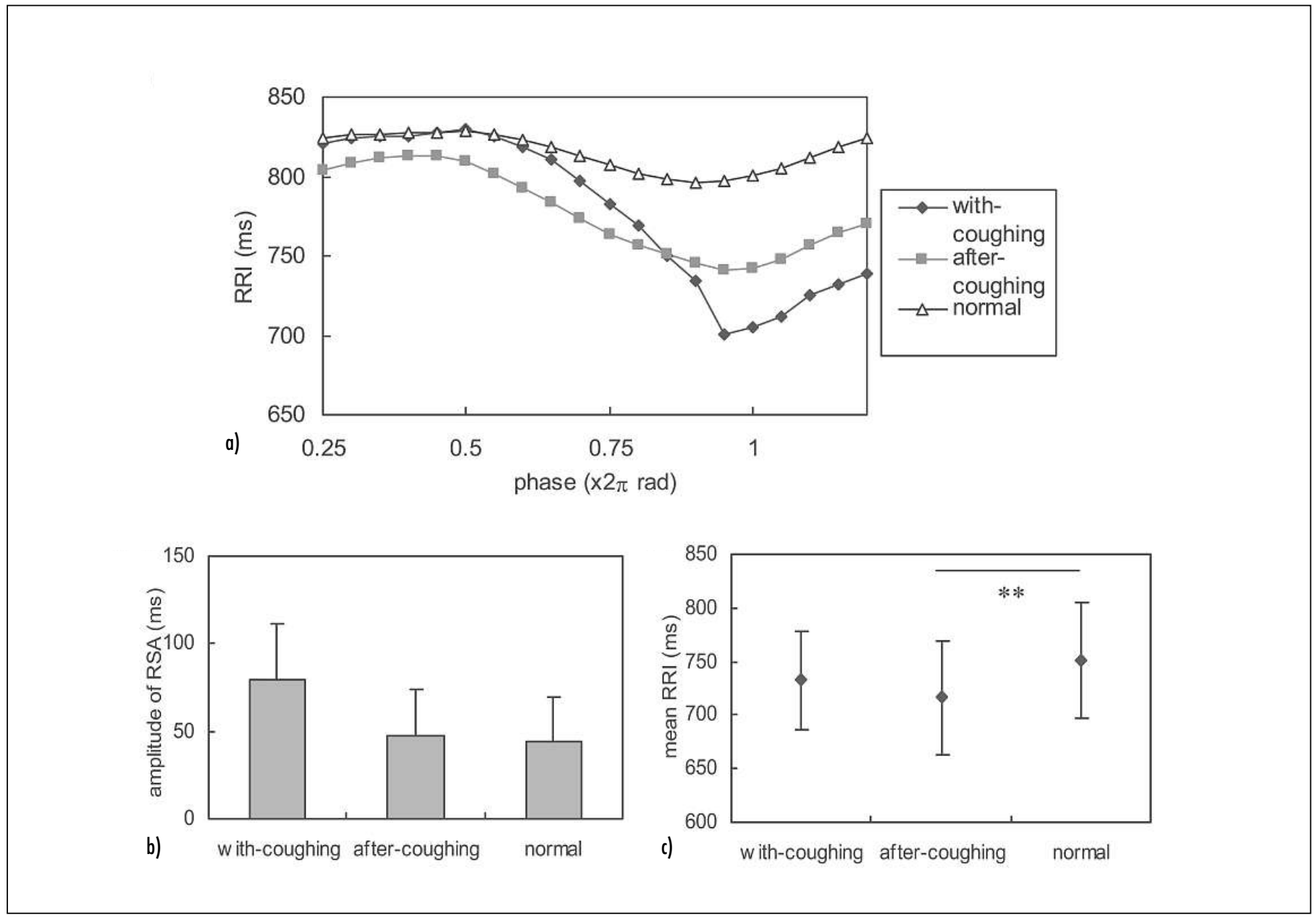

Fig. 4 Results of Exp. 2. a) RSA waveforms of a typical subject, b) RSA amplitudes of all subjects, c) mean RRIs of all subjects. ${ }^{* *}$ indicates $P<0.01$.

difference between the maximum and minimum of the RSA waveform. The statistical difference was tested by repeated measures ANOVA with a post hoc Bonferroni correction in Exps. 1 and 2, and by paired t-test in Exp. 3. In all the experiments, both the first and last $20 \mathrm{~s}$ were eliminated by filtering, and the remaining part was used for analysis.

\section{Results}

\subsection{Influence of Swallowing}

Physiologically, swallowing generates a sudden change in intrathoracic pressure. In Exp. 1-1, all swallowing is observed after expiration (i.e. around $\pi \mathrm{rad}$ in the respira- tory phase), and the numbers of swallows in Exps. 1-1 and 1-2 were $6.2 \pm 1.5$ and $8.0 \pm 4.0$, respectively. Figure 2 shows the results of Exp. 1-1. By RSA waveform, it can be seen that swallowing induces tachycardia and that after-swallowing respiration has almost the same waveform as normal respiration (Fig. 2a). The comparison between the amplitude of RSA with swallowing and that of normal respiration is statistically significant $(P<0.01)$. The amplitude of RSA after swallowing, however, shows no statistically significant difference when compared with normal respiration (Fig. 2b). These results show that swallowing causes tachycardia, but that the tachycardia recovers quickly. The mean RRI of withswallowing respiration has a lower value because of tachycardia $(P<0.01)$, but that of after-swallowing respiration shows no sig- nificant difference from normal respiration (Fig. 2c).

Figure 3 shows the results of Exp. 1-2. It can be seen that swallowing induces tachycardia around $2 \pi \mathrm{rad}$ in the respiratory phase and that recovery occurs after one respiration (Fig. 3a). Therefore, the same statistical features as in Exp. 1-1 are observed in the results of RSA amplitude (Fig. 3b), though the results of mean RRI are different because the influence of tachycardia is still evident at the beginning of the afterswallowing respiration (Fig. 3c).

\subsection{Influence of Coughing}

Coughing also generates a sudden change in intrathoracic pressure, but it is associated with strong inspiration and expiration. After 


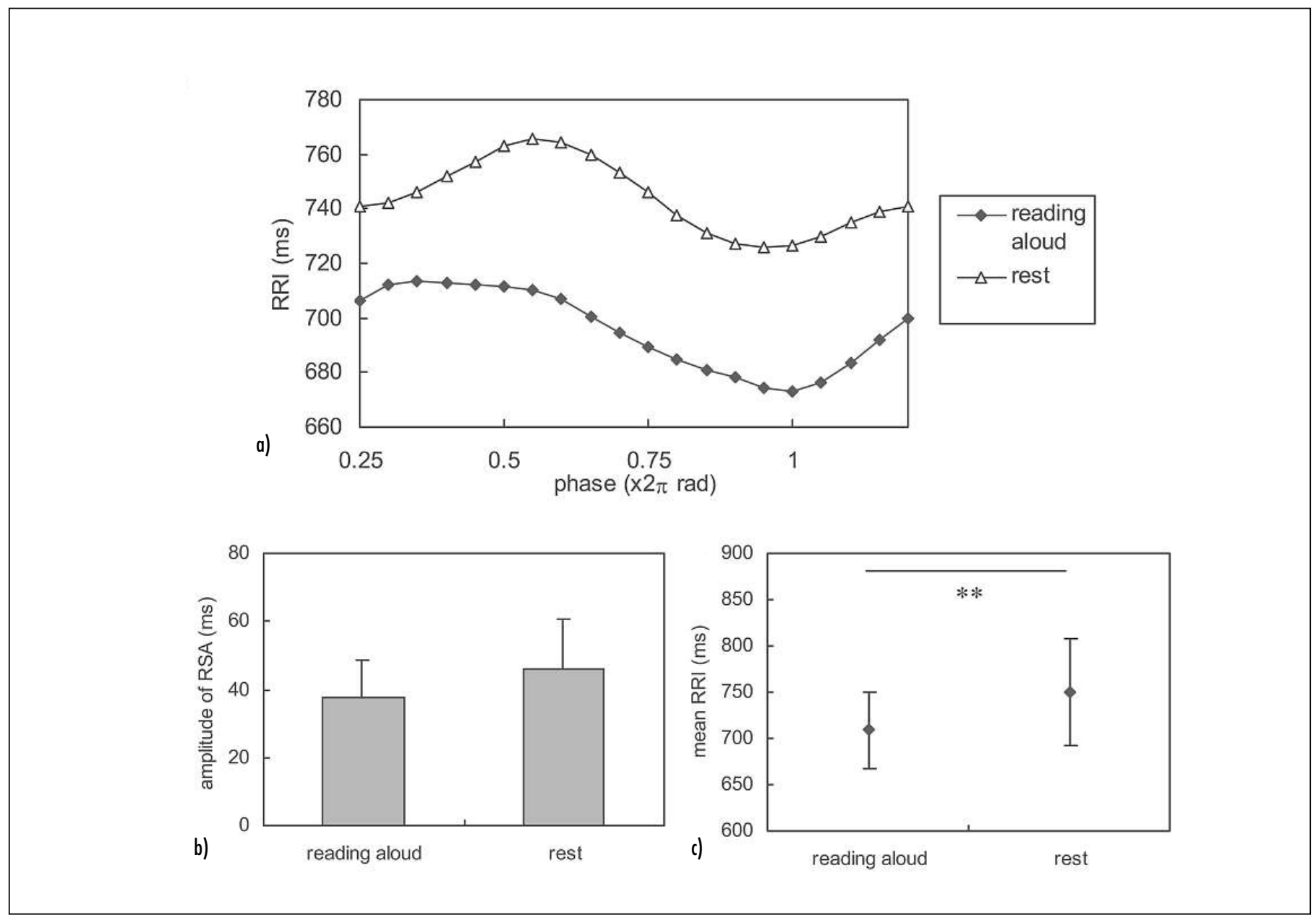

Fig. 5 Results of Exp. 3-1. a) RSA waveforms of a typical subject, b) RSA amplitudes of all subjects, c) mean RRIs of all subjects. ${ }^{* *}$ indicates $P<0.01$.

coughing, if there is disturbed respiration (such as rapid inspiration) considered to be the effect of the previous coughing, these data are discarded and the first normal respiration is selected as the after-coughing respiration. Figure 4 shows the results of Exp. 2. Tachycardia caused by rapid inspiration and the change in intrathoracic pressure is observed in the RSA waveform of coughing, and this tachycardia does not recover within one respiration (Fig. 4a). Therefore, the mean RRI of after-coughing respiration is shorter than normal respiration $(P<0.01)$ (Fig. 4c). The amplitude of RSA with coughing is large but not statistically significant because the magnitude of coughing differs between individuals (Fig. 4b).

\subsection{Influence of Vocalization}

During vocalization, respiration is composed of short inspiration and long expiration. Reading aloud continues its state, while conversation has some silent (listening) periods. Figure 5 and Figure 6 (a-c) show the results of Exps. 3-1 and 3-2, respectively. These results are almost the same, and the mean RRI of vocalization is shorter than in the rest condition and is statistically significant $(\mathrm{P}<0.01$ in Fig. $5 \mathrm{c}$ and $\mathrm{P}<0.05$ in Fig. 6c). However, a non-significant difference is found between the amplitude of RSA in vocalization and rest conditions (Fig. 5b and Fig. 6b). In order to investigate whether the differences appeared in the short-term transition from vocalizing to silence, the waveform of conversation was divided into speaking and listening, and these were compared. The data of one subject who had almost no listening period was discarded and the data of the remaining six subjects were analyzed. The results are shown in Figure 6 (d and e). The mean RRI of speaking is shorter than that of listening, but the difference is not statistically significant.

\section{Discussion}

In Exps. 1-1 and 1-2, swallowing-induced tachycardia is observed and is thought to be caused by a sudden change in intrathoracic pressure. The change in intrathoracic pressure affects venous return and blood pressure, and these in turn change the heart rate by the Bainbridge reflex and baroreflex, re- 

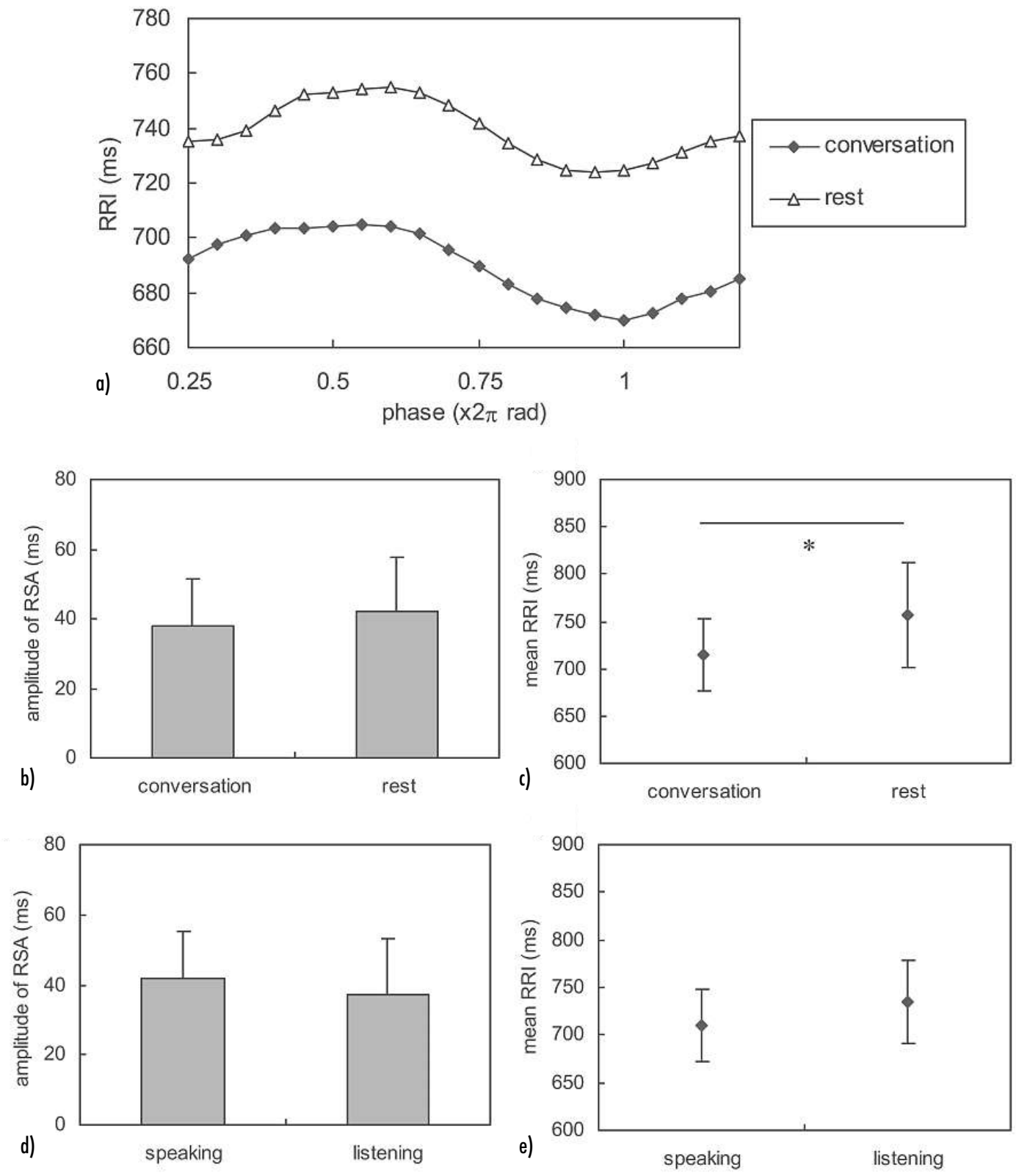

Fig. 6 Results of Exp. 3-2. a) RSA waveforms of a typical subject, b) RSA amplitudes of all subjects, c) mean RRIs of all subjects, d) RSA amplitudes for speaking and listening during conversation, e) mean RRIs of speaking and listening during conversation. ${ }^{*}$ indicates $P<0.05$.

spectively. It is found that this tachycardia recovers within one respiration. Swallowing-induced tachycardia shows a rapid change and disguises the RSA extraction by usual frequency analysis. Coughing is also associated with a sudden change in intrathoracic pressure. However, it requires stronger activity of the respiratory organs and results in short mean RRI of after-coughing respiration. In the cases of vocalization, a short mean RRI is observed, and this may be caused by the activity of vocalization (i.e. sympathetic nervous activity increases and vagal activity decreases). Changing the respiratory pattern by vocalization has no statistically significant influence on the RSA amplitude. Therefore, for the purpose of accurate extraction of the RSA amplitude, the respiration with swallowing must be discarded and the respiration with coughing must also be discarded for certainty.

As for the signal-processing algorithm proposed in this study, it is found that this method is effective for analysing the extrin- 
sic influence on HRV, and it can detect even the short-term influence that is hidden by RSA. The same procedure can be adapted for precise investigation of other influences such as visual or auditory stimulus on HRV by stimulating in the same respiratory phase.

Recently, the complexity of healthy human HRV has been investigated in the sense of statistical physics, and interesting features such as multifractality [8] and a non-Gaussian scale invariant property [9] have been observed. But the mechanism of these features is not fully understood. Therefore, investigation of how HRV reflects extrinsic elements is also important for physical science. For further understanding of these findings, it would be necessary to study the effects of swallowing, coughing and vocalization in detail, because they would appear even during constant routine protocols [10]. The results of this study indicate that swallowing may result in strong singularity, and coughing may result in relatively weak singularity. Vocalization may also result in relatively weak singularity, and its strength depends on the length and timing of the vocalization.

\section{Conclusion}

We have investigated the influence of swallowing, coughing and vocalization on HRV using respiratory-phase domain analysis. The results show that swallowing induces rapid tachycardia that recovers quickly, while tachycardia of coughing continues in after-respiration. In the case of vocalization, the mean RRI becomes short because of the activity, but the amplitude of RSA shows no statistical difference, indicating that the respiratory pattern of vocalization has no influence on RSA amplitude.

\section{Acknowledgments}

The authors wish to thank K. Furukawa for supporting the experiments and Dr. M. Imran Al-Haq for improving the manuscript. This research is partly supported by the 21 st century COE programme, "Information Science and Technology Strategic Core", of the Ministry of Education, Culture, Sports, Science and Technology of Japan.

\section{References}

1. Task Force of the European Society of Cardiology and the North American Society of Pacing and Electrophysiology. Heart rate variability: standards of measurement, physiological interpretation and clinical use. Circulation 1996; 93: 1043-1065.

2. Katona PG., Jih F. Respiratory sinus arrhythmia: noninvasive measure of parasympathetic cardiac control. J Appl Physiol 1975; 39: 801-805.

3. Pomeranz B. Assesment of autonomic function in humans by heart rate spectral analysis. Am J Physiol 1985; 248: H151-153.

4. Kotani K, Hidaka I, Yamamoto Y, Ozono S. Analysis of respiratory sinus arrhythmia with respect to respiratory phase. Methods Inf Med 2000; 39: 153-156.
5. Kotani K, Tachibana M, Takamasu K. Repiratory phase domain analysis can estimate cardiac vagal activity accurately during a mental arithmetic task. Methods Inf Med. In press.

6. Han K, Nagel JH, Schneiderman N. A continuous representation of heart rate. Proc Ann Int Conf IEEE/EMBS 1992; 14: 785-786.

7. Rosenblum MG. Phase synchronization of chaotic oscillators. Phys Rev Lett 1996; 76 : 1804-1807.

8. Ivanov PC. Multifractality in human heartbeat dynamics. Nature 1999; 399: 461-465.

9. Kiyono K, Struzik ZR, Aoyagi N, Sakata S, Hayano J, Yamamoto Y. Critical scale-invariance in healthy human heart rate. Phys Rev Lett 2004; 93 : 178103-1-4.

10. Aoyagi N, Ohashi K, Yamamoto Y. Frequency characteristics of long-term heart rate variability during constant routine protocol. Am J Phys 2003; 285: R171-176.

Correspondence to:

K. Kotani, Ph.D.

Graduate School of Information Science and Technology

The University of Tokyo

7-3-1 Hongo, Bunkyo-ku

Tokyo 113-8656

Japan

E-mail:kota@nano.pe.u-tokyo.ac.jp 